\title{
REAL-TIME BLOB-WISE SUGAR BEETS VS WEEDS CLASSIFICATION FOR MONITORING FIELDS USING CONVOLUTIONAL NEURAL NETWORKS
}

\author{
Andres Milioto, Philipp Lottes, Cyrill Stachniss \\ Institute of Geodesy and GeoInformation, University of Bonn, Germany \\ (andres.milioto,philipp.lottes,cyrill.stachniss)@igg.uni-bonn.de
}

KEY WORDS: Agriculture Robotics, Convolutional Neural Networks, Deep Learning, Computer Vision, Unmanned Aerial Vehicles

\begin{abstract}
:
UAVs are becoming an important tool for field monitoring and precision farming. A prerequisite for observing and analyzing fields is the ability to identify crops and weeds from image data. In this paper, we address the problem of detecting the sugar beet plants and weeds in the field based solely on image data. We propose a system that combines vegetation detection and deep learning to obtain a high-quality classification of the vegetation in the field into value crops and weeds. We implemented and thoroughly evaluated our system on image data collected from different sugar beet fields and illustrate that our approach allows for accurately identifying the weeds on the field.
\end{abstract}

\section{INTRODUCTION}

Agrochemicals can have several side-effects on the biotic and abiotic environment and may even harm human health. Therefore, we need better tools and novel approaches to achieve a reduction in the use of agrochemicals in our fields. In conventional weed control, the whole field is treated uniformly with a single herbicide dose, spraying the soil, crops, and weed in the same way. In order to perform selective spraying, we must acquire knowledge about the spatial distribution of the weeds and plants in the field. One way to collect this data is through UAVs equipped with vision sensors in combination with an automatic classification system that can analyze the image data and label the crops as well as the weeds in the acquired images. One could even go a step further and use autonomous ground vehicles to execute a mechanical or laser-based elimination of the individual weed plants identified by the UAV.

In this paper, we address the problem of classifying image content into crops and weeds. By exploiting the georeferenced location of the UAV, the location for each plant can be determined using a bundle adjustment procedure. Through a detection on a per-plant basis of sugar beets (crops) and typical weeds, we can select relevant information for the farmers or for instructing an agricultural ground robot operating on the field. Given the 3D information and a pixel-wise labeling of the images obtained with our approach, we can directly provide the location of every weed to a ground robot or to a selective spraying tool used on a traditional GNSS-controlled tractor.

Thus, we focus in this paper on the vision-based classification system for identifying sugar beet plants and weeds. We rely on RGB and near infra-red (NIR) imagery of agricultural fields (see Figure 1 for an example). We use modern convolutional neural networks and deep learning pipelines to separate the plants based on their appearance. Our approach is an end-to-end approach, i.e., there is no need to define features for the classification task by hand.

The main contribution of this paper is a vision-based classification system to separate value crops from weeds using convolutional neural networks (CNNs). We target sugar beets as crops,
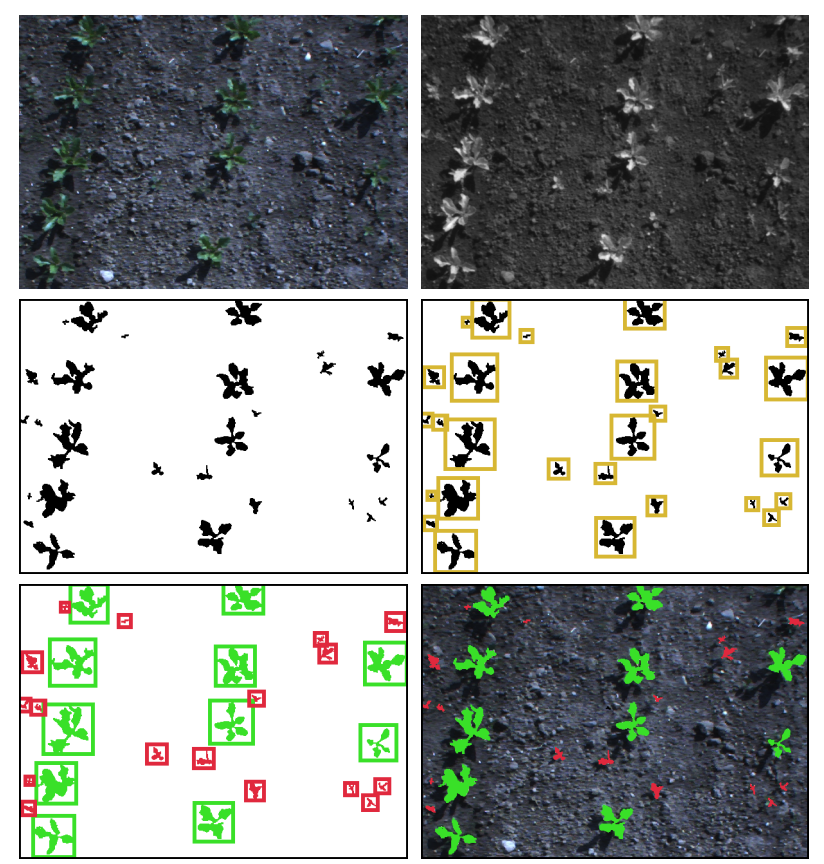

Figure 1. Illustration of our approach. Top: Original RGB and NIR images. Middle: NDVI mask and connected blob segmentation. Bottom: Classified blobs and pixel-wise overlay to original image.

which is an important crop in Northern Europe. To build up our classification system we provide an end-to-end approach where no hand-crafted features are used, as done in most of the previous work on this topic (Lottes et al., 2016a; Wang et al., 2008; Kumar et al., 2012; Cerutti et al., 2013; Elhariri et al., 2014; Perez-Ortiz et al., 2016). The only preprocessing step we perform separates vegetation from background such as soil, etc. We then only focus on the image regions containing vegetation. These sub-images are scaled to a standard size and fed into the neural network. As we show in this paper, there is no need for the network to be especially deep to provide high-quality classification results for this task, and as a result, the amount of training data that is required to train the $\mathrm{CNN}$ is comparably small, in the order of a few hundred 
images. Furthermore, the network can be executed in real-time in a UAV.

In sum, we make the following four key claims: Our approach (i) does not require any hand-crafted features and thus can be implemented quickly, (ii) is able to provide accurate weed classification in real sugar beet fields with an accuracy of more than $95 \%$ in a field with plants in similar growth stage, (iii) can be retrained efficiently to reuse the learned features for other data types or later growth stages, and (iv) is lightweight and fast, which makes it suitable for online operation in a flying robot.

\section{RELATED WORK}

The ability to estimate semantic information from sensor data is an important capability for autonomous and semi-autonomous systems operating indoors (Stachniss et al., 2005) as well as outdoors (Lottes et al., 2016a). For example, mobile robots like UAVs and ground vehicles serve as sensor platforms to obtain fast and detailed information of arable field environments. Robotic approaches for monitoring key indicators of crop health have become a popular research topic. Geipel et al. (2014), Khanna et al. (2015), and Pfeifer et al. (2016) estimate the crop height as well as the canopy cover using UAV imagery. These works rely on bundle adjustment procedures to compute terrain models and subsequently perform a vegetation segmentation to estimate the crop height based on the obtained 3D information.

Several approaches have been developed for vision-based vegetation detection by using RGB as well as multispectral imagery of agricultural fields (Guo et al., 2013; Hamuda et al., 2016; TorresSanchez et al., 2015). Hamuda et al. (2016) present a survey about plant segmentation in field images by analyzing several threshold based as well as learning based methods. Torres-Sanchez et al. (2015) investigate an automatic and adaptive thresholding method for vegetation detection based on the Normalized Difference Vegetation Index (NDVI) and the Excess Green Index (ExG). They report a vegetation detection rate of around $90-100 \%$. In contrast, Guo et al. (2013) apply a learning-based approach using a decision tree classifier for vegetation detection. They exploit spectral features using different color spaces based on RGB images to perform a prediction on a per-pixel basis.

In the context of leaf image classification and segmentation, researchers have investigated using hand-crafted features and structural operators (Wang et al., 2008; Kumar et al., 2012; Cerutti et al., 2013; Elhariri et al., 2014). Wang et al. (2008) segment leaf images by using morphological operators and shape features and apply a moving center hypersphere classifier to infer the plant species. Kumar et al. (2012) start from segmented images of leaves. They extract curvature features and compare them with a given database to find the best match with a labeled type. To cover a variety of leaf shapes, Cerutti et al. (2013) apply a deformable leaf model and use morphological descriptors in order to classify their species. Elhariri et al. (2014) compare a Random Forest classifier and a linear discriminant analysis based approach in their work for classifying 15 plant species by analyzing leaf images. They extract statistical features from the HSV color space of leaf images as well as gray level co-occurrence matrices to exploit additionally shape and vein (leaf structure) information. Hall et al. (2015) conducted a study on features for leaf classification. They compared the classification performance based on classical hand-crafted and ConvNet features by using a random forest classifier. They simulated varying conditions on the public
Flavia leaf dataset and conclude that ConvNet features support the performance and robustness of the classification results.

In contrast, we avoid the time consuming process of designing features by hand and exploit a CNN approach in order to completely learn a representation of the data, which is suitable for discriminating sugar beets and weeds in image patches.

In the field of machine learning, several approaches have been applied to classify crops and weeds in imagery of plantation (GarciaRuiz et al., 2015; Guerrero et al., 2012; Perez-Ortiz et al., 2016, 2015; Haug et al., 2014; Lottes et al., 2016a, 2017; Latte et al., 2015). Perez-Ortiz et al. (2015) propose an image patch based weed detection system. They use pixel intensities of multispectral images and geometric information about crop rows in order to build features for the classification. They achieve overall accuracies of $75-87 \%$ for the classification by analyzing the performance of different machine learning algorithms. Perez-Ortiz et al. (2016) use a support vector machine classifier for crop/weed detection in RGB images of sunflower and maize fields. They exploit statistics of pixel intensities, textures, shape and geometrical information as features. Guerrero et al. (2012) propose a weed detection approach for image data containing maize fields. Their method allows to identify the weeds after its visual appearance changed in image space due to rainfall, dry spell or herbicide treatment. Garcia-Ruiz et al. (2015) propose an approach for separating sugar beets and thistle based on narrow band multispectral image data. They apply a partial least squares discriminant analysis for the classification and obtain a recall of $84 \%$ for beet and $93 \%$ for thistle by using only four of the narrow bands at 521, 570, 610 and $658 \mathrm{~nm}$. Haug et al. (2014) present a method to detect carrot plants in weeds by using RGB and NIR-images without needing a presegmentation of the scenes into vegetation blobs. They perform a prediction on pixel level on a sparse grid in image space and report an average accuracy of around 94\% on an evaluation set of 70 images where both, intra and interrow overlap is present. In previous work, Lottes et al. (2016a, 2017) design a crop and weed classification system for ground and aerial robots. The work exploits NDVI and ExG indices to first segment the vegetation in the image data and then applied a random forest classifier to the vegetative parts to further distinguish them into crops and weeds. Latte et al. (2015) use color space features and co-occurrence matrices to classify images captured on crop fields containing several plant types. They apply an artificial neural network classifier and obtain an accuracy around $84 \%$ on average. Their results show that using statistical moments of the HSV distribution improves the overall classification performance.

Also deep learning methods have recently been employed to obtain plant statistics and distinguish value crops and weeds in the fields (Mortensen et al., 2016; Chen et al., 2017; McCool et al., 2017; Potena et al., 2016). Mortensen et al. (2016) apply a deep $\mathrm{CNN}$ for classifying different types of crops to estimate individual biomass amounts. They use RGB images of field plots captured at $3 \mathrm{~m}$ above soil and report an overall accuracy of $80 \%$ evaluated on a per-pixel basis. Chen et al. (2017) propose a visual system with the purpose of obtaining a robust count of fruits in the field under dramatic lighting changes, and with heavy occlusions from neighboring foliage. They first use a CNN for blob region proposal, then they use a counting algorithm based on a second $\mathrm{CNN}$ to estimate the fruit count in each region, and they finally apply a linear regression model to get the final count. McCool et al. (2017) address the crop and weed segmentation 
problem by using an end-to-end ensemble of CNNs. They obtain these lightweight $\mathrm{CNNs}$ through the compression of a pretrained, more descriptive and complex model, and report an accuracy of $93.9 \%$, processing images at little over 1 frame per second. Potena et al. (2016) present a perception system based on RGB+NIR imagery for crop and weed classification using two different CNN architectures. A shallow network performs the vegetation detection and then a deeper network further distinguishes the detected vegetation into crops and weeds. They perform a pixel-wise classification followed by a voting scheme to obtain predictions for detected blobs in the vegetation mask. They obtain a performance of around $97 \%$ for the vegetation detection, which is comparable to a threshold based approach based on the NDVI, and $98 \%$ for the crop and weed classification in case the visual appearance has not changed between training and testing phase.

Our approach uses RGB-NIR imagery and CNNs, and is able to reach an accuracy of $97 \%$ with low running times even in embedded hardware that can be fitted in a flying platform. We achieve 97\% precision and 98\% recall for the classification of the weeds when the classifier is used in a similar growth stage as the one it was trained in. We are also able to obtain $99 \%$ recall and $89 \%$ precision for weeds in later growth stages without retraining. Finally, we show that we can jump from $89 \%$ to $97 \%$ in recall for weeds if we retrain the classifier with new data from the current growth stage in a fast way. All of this allows our system to do statistics and selective weed removal in a very quick and accurate manner.

\section{VISION BASED PLANT AND WEED CLASSIFICATION}

The main goal of our plant classification pipeline is to allow mobile agricultural robots to detect unwanted species to remove them reactively, by either applying selective spraying or mechanical extraction/destruction. This requires the algorithm to run in realtime (or near real-time) on hardware that is compact and light enough to run in a flying platform, which is our principal aim. In our case, we consider the case of sugar beet plantations, which is a valuable study case both due to its popularity in Northern Europe, and because it has been solved before using other approaches, which gives us the possibility to compare accuracy, robustness and real-time capabilities against other state-of-the-art algorithms.

We need to achieve a high recall on the weed classification in order to enable the robot to remove a high number of weeds on the field. Therefore, we detect the coordinates relative to the robot's coordinate system to synchronize the removal with the extraction/spraying systems. This means that we are relaxed on the boundaries of the plants as long as we are able to direct the removal tools to the right position within our desired accuracy without harming the valuable crops. This allows for certain hypotheses that make a computationally cheap and fast blob approach suitable while still achieving state-of-the-art performance, such as: doing most of the training of the classifier and weed removal in early stages of the crop growth, and relaxing on the precision of the boundaries of the vegetation at the pixel level. However, as we can see in the last image of Figure 1, where the output classification is overlayed with the input image, a combination of the NDVI thresholded segmentation with a blob segmentation and classification gives a solid almost pixel-wise representation of the output mask, due to the masking done by the preprocessing, making this algorithm not only faster but also very similar in
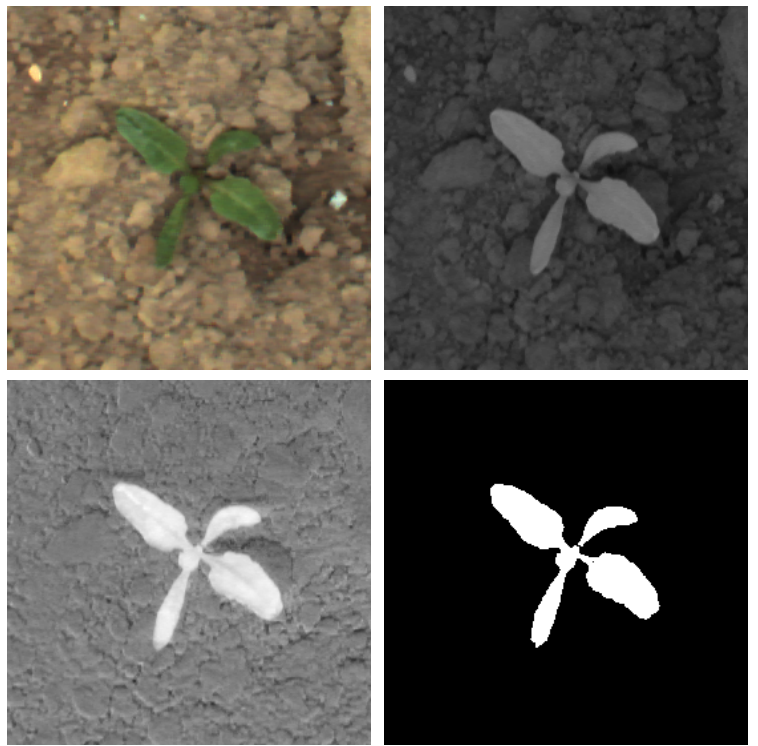

Figure 2. Top: RGB and NIR images. Bottom: NDVI transform and mask obtained by a conservative threshold in the NDVI image.

output quality as the pixel-wise approach, which is both computationally more complex and more time expensive to label.

We divide our classification pipeline in three main steps. First, we perform a vegetation detection to eliminate irrelevant soil information from the images using multispectral images containing RGB and near infra-red information. Second, we describe the detected vegetation with a binary mask and perform a blob segmentation in order to extract patches containing singular crops or weeds. Finally, we classify each patch with a convolutional neural network that we train in an early growth stage to avoid overlapping crops and weeds in the data, and that we can later on re-train in a supervised or semi-supervised manner to adapt to other types of crops, or other stages of the crop growth. The blob approach makes the network run fast for both the forward pass and the retraining of the classifier, which makes it able to run on a flying platform using readily available single-board computers such as the NVIDIA Jetson platform. In the following subsections we discuss each step of the pipeline in more detail.

\section{Vegetation Detection}

The goal of this pre-processing step is to separate the relevant vegetation content of every image $\mathcal{I}$ taken by the robot from the soil beneath it, for which we need to apply a mask of the form:

$$
\mathcal{I}_{\mathcal{V}}(i, j)=\left\{\begin{array}{ll}
1, & \text { if } \mathcal{I}(i, j) \in \text { vegetation } \\
0, & \text { otherwise }
\end{array},\right.
$$

with the pixel location $(i, j)$.

As we can see in Figure 2, separating vegetation from soil in a plain RGB image is difficult task even for a human observer. Luckily, an approach exploiting the high reflectance of chlorophyll in the near infra-red spectrum (NIR) has been thoroughly studied by Rouse et al. (1974) prompting us to use the Normalized Difference Vegetation Index (NDVI), which is a normalized ratio of the NIR and red bands. If we apply this index pixelwise we obtain a distribution that is more suited for a thresholded segmentation than the original image, which we can later apply 
as our mask, as we can also see in Figure 2. Namely, using the NDVI to create the mask we have:

$$
\mathcal{I}_{\mathrm{NDVI}}(i, j)=\frac{\mathcal{I}_{\mathrm{NIR}}(i, j)-\mathcal{I}_{\mathrm{R}}(i, j)}{\mathcal{I}_{\mathrm{NIR}}(i, j)+\mathcal{I}_{\mathrm{R}}(i, j)}
$$

Such transformation is used successfully to solve this very problem by Lottes et al. (2016b), and improved by Potena et al. (2016) by adding a low cost convolutional neural network to a mapping obtained by a very permissive thresholding in the post NDVI segmentation operation. In our experiments, we use the thresholded segmentation of the NDVI-transformed image, similar to Lottes et al. (2016b), to separate the plants from the soil. An example is shown in Figure 2.

A threshold-based classification based on the NDVI can also lead to individual pixel outliers (or small groups of pixels). These are mostly caused by lens errors as chromatic aberration, which leads to a slightly different mapping of the red and the near infrared light on the camera chip. We eliminate most of these effects through basic image processing techniques such as: morphological opening and closing operations to fill gaps and to remove noise at contours, which is done with a $5 \times 5$ disk kernel $1 \times$ opening followed by a $1 \times$ closing; and removal of tiny blobs which share less than a minimum amount of vegetation pixels, which depends on the Ground Sampling Distance and therefore it varies with the UAV distance to ground.

\section{Blob-wise Segmentation}

Given the vegetation mask we search for vegetation blobs. Each vegetation blob is given through a connected component of the vegetation pixels in the image (see Figure 3 ). We create an individual image patch of a certain size $(64 \times 64$ pixels in our current setup) for each detected blob in the vegetation mask. Here, we first compute the bounding box of the contour of the blob and apply an affine transformation to it in order to translate the blob into the center of the image patch and scale it to the desired size.

These image patches serve as input for our classification approach Thus, the CNN can learn a representation based on such patches, which mostly describe whole plants or leaves. Through centering the blobs within the image patches we achieve translation invariance and through scaling the detected blobs we reduce the influence of the plant size, which can vary a lot even for plants growing on the same field.

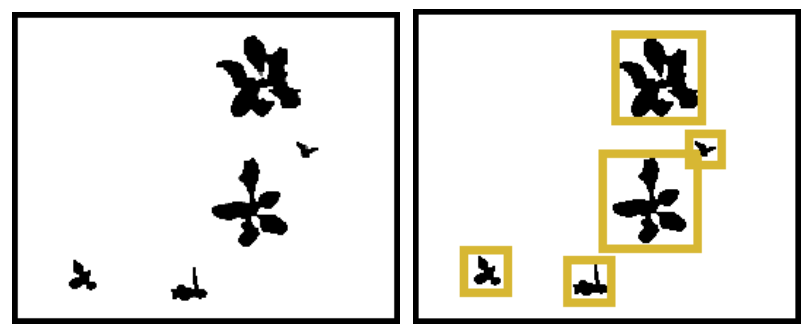

Figure 3. Blob selection from connected regions.

\section{Classification using Convolutional Neural Networks}

The advantage of using Convolutional Neural Networks for image classification is that they have the ability to learn features describing complex non-linear relations between dependent and independent variables by themselves, when given enough data.
This is not only useful for achieving a high classification accuracy in a specific dataset, but also to later transfer that knowledge to other domains. Therefore, we can train the network with a high number of plants from different species under different weather and lighting conditions and then use the feature extractors to cheaply train other classifiers for unseen species.

The main ideas behind weight sharing and CNNs were introduced in LeCun et al. (1989) and LeCun et al. (1998), and more recently brought back to public interest by Krizhevsky et al. (2012) where they exploited an efficient GPU implementation of the convolution to break the ImageNet challenge benchmark at the time by an astounding $10 \%$ difference over their closest competitor, making them widely popular. They make the assumption that in image data, neighboring pixels are more correlated than pixels far away, therefore introducing the concept of local connectivity, allowing for less neuron wiring, which yields not only in better results, but also in less training and execution time. Today, deep convolutional neural networks are undoubtedly the most widely used approach for image classification, with it's more novel flavors achieving top-5 classification errors in the test set of ImageNet as low as $3.57 \%$ (He et al., 2015), and 3.08\% (Szegedy et al., 2016), outperforming the average human classification error which is around $5 \%$.

The original architecture used by Krizhevsky et al. (2012) is comparably shallow when measured against today's state-of-the-art image classification networks, but we use a similar one to solve our classification problem, because it proves to have enough expressiveness to describe our decision boundaries and has the advantage that it is cheap to train and run in compact and low-power consumption hardware such as the one in a UAV.

\section{Selection of the Architecture}

The network has to be expressive enough to be able to describe the decision boundary between classes, but not too expressive as to overfit to our training data. This characteristic is set by the depth of the network, the amount of kernels in each convolutional layer and the amount of neurons in the fully connected ones. To choose our network size, we iterate starting with networks of a small number of filters per layer and of only one convolutional layer and one fully connected, and grow the network until we are almost able to overfit to a small sample of our dataset. After this, we further increase the expressiveness by adding more kernels to each convolutional layer and more neurons to the fully connected ones, and we train it again using our full training dataset. To avoid the bigger network to overfit to our training data we use a state-of-the-art method called "dropout" (Srivastava et al., 2014). This prevents neurons from learning complex co-adaptations in the fully connected layers by randomly dropping some of them during training, making the network generalize better, which we corroborate by getting a good performance in our cross-validation data. We also have to be careful to set an upper boundary in the network's complexity, due to our constraint of real-time capability, meaning that the network needs to be both lightweight and of a complexity that is low enough to run fast in an embedded platform that we can fit in a flying vehicle. We are careful when choosing the depth and size of each layer to enforce this restriction.

Finally, we want our classifier to represent a vector containing the a probablity distribution of the input patch being a plant and a weed. For this, we use output neurons with a softmax activation 


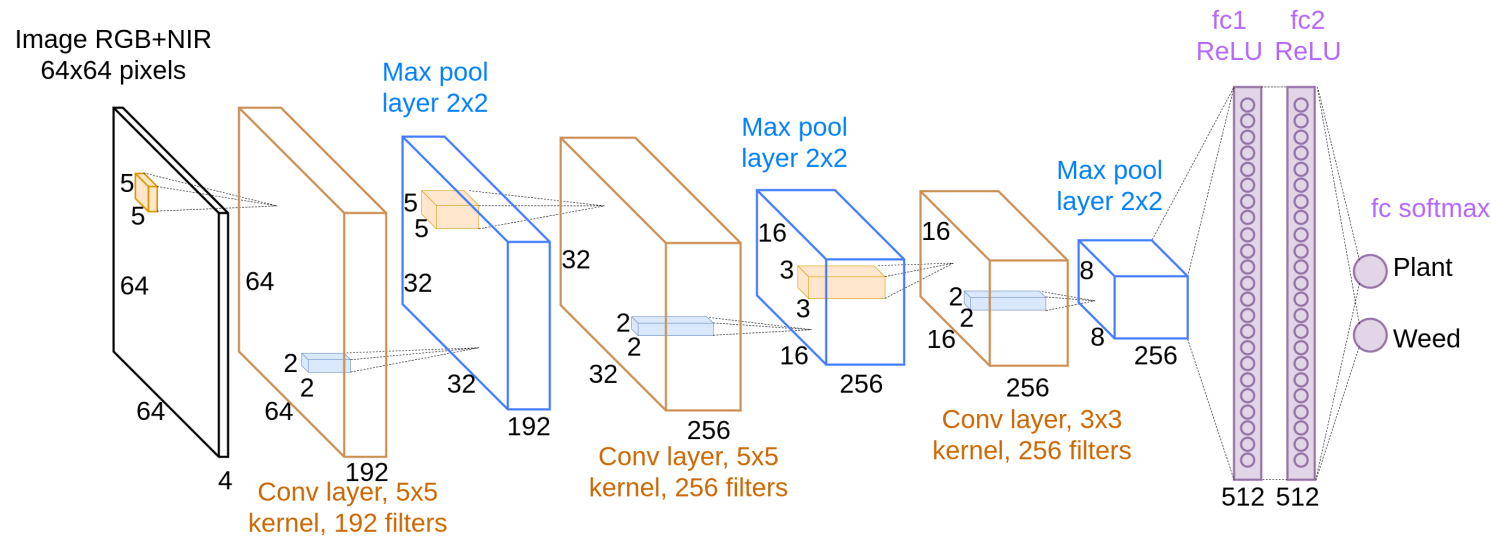

Figure 4. Detailed architecture used for the blob-wise classification.

function, which convert the output of the network to a pseudoprobability distribution that works well for this problematic.

From this we design a network like the one in Figure 4. This architecture is composed by 3 convolutional layers ( 2 of kernel size $5 \times 5$ and the last one of $3 \times 3$ kernels), all of them using the Rectified Linear Unit for the non-linearity, which proves to speed up training considerably, and followed by $2 \times 2$ max-pooling for dimensionality reduction. These 3 convolutional and max pooling layers are followed by 2 fully connected layers with 512 neurons each, which results in a 512-dimensional vector to be fed into the softmax layer that outputs the pseudo probability distribution we want as an output.

It is important to note that even though the network is shallow compared to the ones used by He et al. (2015); Szegedy et al. (2016), it is more complex and than those normally used for plant discrimination problems (Potena et al., 2016). However, due to the blob approach, the network doesn't need to examine every pixel of the original input image, and it only runs one time per segmented patch, speeding up the process considerably.

\section{Retraining for different crop growth stage}

As the plants grow, they not only change their size, but also their shapes and colors, which means that we may not get the best results if the growth stage is very different. In this case, the features extracted from the convolutional and fully connected layers may still be useful, so we can try to retrain the last layer of the network, which is the classifier, by feeding the network with a sample of labeled data in the new growth stage. We will show that even though the classifier is very good without retraining, this technique can yield significant improvements, and can be done in a fast way due to the need to only train a linear classifier and in a small subset of the new data.

\section{EXPERIMENTAL EVALUATION}

The experiments are designed to show the accuracy and efficiency of our method and to support the claims made in the introduction that our classifier is able to (i) learn feature representations by itself and thus be implemented quickly, (ii) learn an accurate decision boundary to accurately discriminate plants and weeds with an accuracy of over $95 \%$, being able to eliminate a majority of weeds with a minimal number of misclassified crops when tested in a similar growth stage; (iii) quickly improve after retraining by using the previously learned features and adapting the last layer to a small subset of labeled new data; and (iv) run in real-time.

We implement the whole approach presented in this paper relying on the Google TensorFlow library. This allows a new $\mathrm{PhD}$ student in the field of plant classification to perform the full implementation of the approach and its evaluation in slightly over two months (not including the acquisition time of the training and evaluation datasets), which supports our claim (i) that due to the self-learning of feature descriptors the pipeline can be implemented quickly.

\section{Training of the Network}

To train the network we use a dataset which we will call "Dataset A", from Table 1. We split the dataset in 3, leaving $70 \%$ of it available to us for training, $15 \%$ for validation, and $15 \%$ for test after the whole pipeline was designed and trained, and therefore show how the network generalizes to unseen data. This dataset was captured with the plants in an early growth stage and it is quite balanced in it's plants/weeds relation. We compare it with Dataset B from Table 1, which was captured 2 weeks later, in a more advanced growth stage, and we analyze the performance of the network.

Both datasets were captured using a JAI camera in nadir view capturing RGB+NIR images. For a typical UAV based monitoring scenario, not every image from the camera needs to be classified, and one image per second is sufficient for real applications. Thus, we take images at $1 \mathrm{~Hz}$, setting the upper bound for testing our real-time classification capabilities to 1 second per raw input image.

\begin{tabular}{r|cc}
\hline Parameter & Dataset A & Dataset B \\
\hline no. images & 867 & 1102 \\
no. patches & 7751 & 28389 \\
no. crops & 2055 & 2226 \\
no. weeds & 5696 & 26163 \\
$P_{c} / P_{w}$ & $0.36 / 0.64$ & $0.08 / 0.92$ \\
avg. patch/image & 9 & 25 \\
\hline
\end{tabular}

Table 1. Information about the datasets.

A commonly used technique for augmenting datasets is to apply affine transformations to the training portion of the dataset, in order for the network to learn some type of invariance. In our case, all the plants are previously scaled to patches of $64 \times 64$ pixels, 
aiming for scale and translation invariance, and we further apply 64 even rotations to try to learn rotationally invariant features. After this we perform a per image normalization, which is also a common practice in learning to speed up the training.

Then we run the training using a cross-entropy cost function, which works well with the ReLU activations, along with a first order optimizer, which we use to minimize the cost by using stochastic gradient descent in batch sizes of 100. During training we reduce the learning rate by a third every 2 epochs to converge better the cost function's minima, and we run until convergence is achieved.

The training takes 3 days in a low-cost NVIDIA GeForce GTX 940MX, which is not a top notch graphics card, and it is actually less powerful than a currently available embedded platform like the Jetson TX2 which runs in a drone. This is critical if retraining is necessary, as we show later. If we use state of the art GPU's for training, the process is cut short by an order of magnitude, not only because of the difference in the floating operations per second, but also because the whole pipeline can be stored in GPU memory avoiding a bottleneck in the copying operations from system memory to GPU memory before each calculation. In Table 2 we show these differences. This further supports our claim (i) that the system can be implemented quickly, given the short training time even in a low-cost GPU.

\begin{tabular}{r|cccc}
\hline Model & Cores & Arch. & TFLOPs & RAM \\
\hline Titan Xp & 3840 & Pascal & 12 & $12 \mathrm{~GB}$ \\
Jetson TX2 SoC & 256 & Pascal & 1.5 & $8 \mathrm{~GB}$ \\
GTX 940MX & 384 & Maxwell & 0.84 & 2GB \\
Intel i7 & - & - & 0.04 & - \\
\hline
\end{tabular}

Table 2. Hardware comparison.

\section{Performance of the Classifier}

This experiment is designed to support our claim (ii) which states that we learn an accurate decision boundary to accurately discriminate plants and weeds with an accuracy of over $95 \%$, being able to eliminate a majority of weeds with a minimal number of misclassified crops.

We now analyze the accuracy of the learned classifier and we evaluate its invariance to the crop growth. We tested the accuracy of our classifier in the test set (which only contains unseen data) of dataset A, and its accuracy in the test set of dataset B, when trained in A. We can see the results in Table 3:

\begin{tabular}{r|cc}
\hline Dataset & Test set A & Test set B \\
\hline Accuracy & $97.3 \%$ & $89.2 \%$ \\
\hline
\end{tabular}

Table 3. Accuracy of the classifier trained in train set of A, and evaluated in Test set of A and B.

As we can see, we have a very high overall accuracy in the unseen data from dataset $\mathrm{A}$, because it is from the same crop growth stage as the data the network has seen while it was being trained, and the performance of the classifier drops when used 2 weeks later. However, the overall accuracy does not say much about the quality of the classifier, especially in cases like dataset B, that are highly unbalanced. Since the main goal of our classifier is to detect weeds to proceed to their elimination, in Table 4 we show the obtained precision and recall for the detection of weeds in both datasets.
It is important to note that due to the nature of our problem it is critical to have a low precision in the weed classifier, because a large number of false positives means that plants get classified as weeds and thus get eliminated, especially in the case of mechanical treatment. This is not the case for selective spraying, but spraying plants with herbicides defeats the purpose of the system anyway. It is also desirable to have a high recall to eliminate the most weeds we can, but not eliminating crops is of a critical importance.

\begin{tabular}{r|cc}
\hline Dataset & Dataset A & Dataset B \\
\hline True Positives & 829 & 16353 \\
True Negatives & 290 & 1406 \\
False Positives & 27 & 94 \\
False Negatives & 16 & 2020 \\
Weed precision & $96.84 \%$ & $99.42 \%$ \\
Weed recall & $98.10 \%$ & $89.00 \%$ \\
\hline
\end{tabular}

Table 4. Quality comparison of the classifier for the weed class trained in A.

Taking this into consideration, we can see from Table 4 that even if the recall of the weed detector decreases two weeks later, the precision is very high, allowing us to not eliminate valuable crops erroneously, fulfilling our demands for the system, and supporting our claim (ii).

\section{Retraining the network}

This experiment is designed to prove claim number (iii) that our system can be easily and cheaply retrained to adapt to new growth stages.

As stated in the previous section, even though the precision of the weed detector was still very high two weeks later in the growth stage, the recall fell considerably, meaning that a good number of weeds were not being removed from the field. To fix this problem, we can cheaply retrain the last layer of the classifier, re-utilizing the feature vectors extracted by the convolutional filters learned from dataset A. We show the results it yields in Table 5, where we can see that both precision and recall have improved, making the system not only eliminate less crops erroneously, but also eliminate more weeds, which is the main purpose of it. In Table 6 we can also assess the general accuracy of the retrained classifier.

\begin{tabular}{r|cc}
\hline Dataset & Trained in A & Retrained in B \\
\hline True Positives & 16353 & 17927 \\
True Negatives & 1406 & 1190 \\
False Positives & 94 & 60 \\
False Negatives & 2020 & 696 \\
Weed precision & $99.42 \%$ & $99.66 \%$ \\
Weed recall & $89.00 \%$ & $96.26 \%$ \\
\hline
\end{tabular}

Table 5. Quality comparison of the classifier for the weed class in test set of $\mathrm{B}$, before and after retraining.

\begin{tabular}{r|cc}
\hline Dataset & Trained in A & Retrained in B \\
\hline Accuracy & $89.2 \%$ & $96.1 \%$ \\
\hline
\end{tabular}

Table 6. Accuracy in dataset test set of B before and after retraining on small subset (15\%) of training set of B.

What is most important to point out is that, while the full classifier takes 3 days to train in the cheap GPU, the retraining achieves remarkable improvements in only 20 minutes. This is done by feeding the training with a balanced sub-sample $(15 \%)$ of dataset $\mathrm{B}$ containing the same number of weeds and plants. 
This supports our claim that our system can be easily and cheaply adapted to new growth stages where plants have significantly changed their appearance.

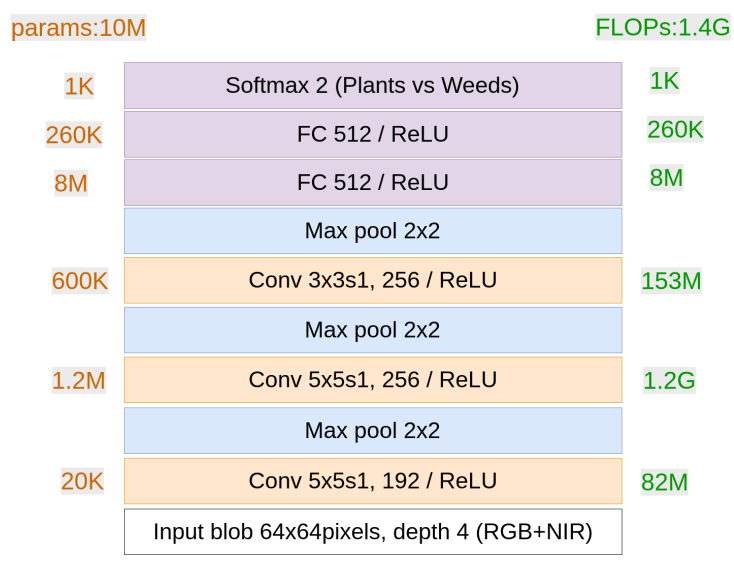

Figure 5. Architecture used for the blob-wise classification, with the size of each layer specified and the amount of floating operations needed per pass.

\section{Runtime}

This experiment is designed to prove claim number (iv) that our system can run in real-time in a flying platform. In Figure 5, we show another representation of the network, in which we can also see how many variables each layer contains, and how many floating operations it takes to pass through it. The final architecture needs $1.4 \mathrm{G}$ floating point operations to do a full forward pass, which means that we can roughly calculate how long it will take to do a full pass depending on the FLOPs of the hardware. This results in Table 7. It is important to note that we left out the most powerful GPU because it is non realistic to think that we would put a desktop workstation in a small flying drone with the purpose of inference.

Another aspect we can see from Figure 5 is that the architecture is using a total number of $10 \mathrm{M}$ 32-bit floating point parameters. This means that we can will have a full network size of $40 \mathrm{MB}$, which is something that our embedded hardware can store easily.

\begin{tabular}{r|cc}
\hline Hardware & Theoretical time & Actual time \\
\hline Jetson TX2 SoC & $1 \mathrm{~ms}$ & $4 \mathrm{~ms} \pm 1 \mathrm{~ms}$ \\
Geforce GTX 940MX & $2 \mathrm{~ms}$ & $7 \mathrm{~ms} \pm 1 \mathrm{~ms}$ \\
i7 Processor & $40 \mathrm{~ms}$ & $50 \mathrm{~ms} \pm 10 \mathrm{~ms}$ \\
\hline
\end{tabular}

Table 7. Average and standard deviation running time per blob, depending on the hardware.

In Table 8, we analyze how long it would take in average to do a forward pass in all patches from a raw image, depending on the dataset. This highly depends on the growth stage, as we can see from the fact that dataset $\mathrm{B}$ has more patches per image, meaning that there are more plants and weeds present in the field, which makes sense. In this table we see that even with 25 images per image, which is our worst case, we are still very relaxed in our real-time constraint, by a margin of 5 in the case of our outdated GPU and a margin of 10 in the state of the art SoC, fulfilling our efficiency claim. This means that we can either analyze images at a higher frame rate, or fly higher, covering more crops, if the resolution of the camera is good enough to capture them from a distance. We also show that for our purposes, it is worth to loose constant time complexity of the algorithm towards linear time because, due to the upper bound of the number of patches contained in each raw image, we are actually gaining in performance.

\begin{tabular}{r|cc}
\hline Dataset & Dataset A & Dataset B \\
\hline avg patches per image & 9 & 25 \\
\hline GEForce940Mx(7 ms/patch avg) & $63 \mathrm{~ms}$ & $175 \mathrm{~ms}$ \\
\hline Jetson TX2 $(4 \mathrm{~ms} /$ patch avg $)$ & $36 \mathrm{~ms}$ & $100 \mathrm{~ms}$ \\
\hline
\end{tabular}

Table 8. Average runtime per image.

As a final comment about runtime, we should consider that for each raw input image containing $n$ vegetation blobs we segment we get a patch array of size $n$. Since our neural network platform allows us to run forward passes in batch, we can gain a considerable increase in performance if we send the whole batch of blobs to the GPU RAM at the same time, rather than queuing all operations sequentially.

The presence of low cost, lightweight, power efficient hardware that can run this classifier in remarkably low time with great performances, make this approach a great alternative for online use in flying platforms working in dynamic environments. This supports our claim number (iv) that our system can run in real-time in a UAV.

\section{CONCLUSIONS}

UAVs as well as other robots for precision farming must be able to distinguish the crops on the field from weeds. In this paper, we addressed the problem of detecting sugar beet plants and weeds using a RGB+NIR camera over real fields. We developed a classification system based on convolutional neural networks (CNNs). Our approach is purely vision-based exploiting 4-channels and does not require geometric priors such as that the sugar beets must been planted in crop rows. In addition to that, the CNNs are an end-to-end approach such that no manual features must be defined. The input to the CNNs are blobs of vegetation and provide a classification output for such blobs. This renders the approach fast such that the system can be executed online. By relying on existing libraries for CNNs such as Tensorflow by Google, our classification system can be replicated quickly in the order of 2 months. We implemented our approach and thoroughly evaluated it using image data recorded on a real farm in different sugar beet fields and illustrate that our approach allows for accurately identifying the weeds on the field.

\section{ACKNOWLEDGMENTS}

This work has partly been supported by the EC under H2020-ICT644227-FLOURISH and by the DFG under FOR 1505: Mapping on Demand. We would like to thank Chebrolu et al. (to appear) for providing part of the data used in our work.

\section{References}

Cerutti, G., Tougne, L., Mille, J., Vacavant, A. and Coquin, D., 2013. A model-based approach for compound leaves understanding and identification. In: Proc. of the IEEE Int. Conf. on Image Processing (ICIP), pp. 1471-1475.

Chebrolu, N., Lottes, P., Schaefer, A., Winterhalter, W., Burgard, W. and Stachniss, C., to appear. Agricultural robot dataset for plant classification, localization and mapping on sugar beet fields. Int. Journal of Robotics Research. 
Chen, S., Skandan, S., Dcunha, S., Das, J., Qu, C., Taylor, C. and Kumar, V., 2017. Counting apples and oranges with deep learning: A data driven approach. IEEE Robotics and Automation Letters.

Elhariri, E., El-Bendary, N. and Hassanien, A. E., 2014. Plant classification system based on leaf features. In: Computer Engineering Systems (ICCES), 2014 9th International Conference on, pp. 271-276.

Garcia-Ruiz, F., Wulfsohn, D. and Rasmussen, J., 2015. Sugar beet (beta vulgaris 1.) and thistle (cirsium arvensis 1.) discrimination based on field spectral data. Biosystems Engineering 139 , pp. 1-15.

Geipel, J., Link, J. and Claupein, W., 2014. Combined spectral and spatial modeling of corn yield based on aerial images and crop surface models acquired with an unmanned aircraft system. Remote Sensing 6(11), pp. 10335.

Guerrero, J. M., Pajares, G., Montalvo, M., Romeo, J. and Guijarro, M., 2012. Support vector machines for crop/weeds identification in maize fields. Expert Systems with Applications 39(12), pp. $11149-11155$.

Guo, W., Rage, U. K. and Ninomiya, S., 2013. Illumination invariant segmentation of vegetation for time series wheat images based on decision tree model. Computers and Electronics in Agriculture 96, pp. 58-66.

Hall, D., McCool, C., Dayoub, F., Sunderhauf, N. and Upcroft, B., 2015. Evaluation of features for leaf classification in challenging conditions. In: Applications of Computer Vision (WACV), 2015 IEEE Winter Conference on, pp. 797-804.

Hamuda, E., Glavin, M. and Jones, E., 2016. A survey of image processing techniques for plant extraction and segmentation in the field. Computers and Electronics in Agriculture 125, pp. 184-199.

Haug, S., Michaels, A., Biber, P. and Ostermann, J., 2014. Plant classification system for crop / weed discrimination without segmentation. In: Proc. of the IEEE Winter Conf. on Applications of Computer Vision (WACV), pp. 1142-1149.

He, K., Zhang, X., Ren, S. and Sun, J., 2015. Deep residual learning for image recognition. Computing Research Repository.

Khanna, R., Möller, M., Pfeifer, J., Liebisch, F., Walter, A. and Siegwart, R., 2015. Beyond point clouds - 3d mapping and field parameter measurements using uavs. In: Proc. of the IEEE Int. Conf. on Emerging Technologies Factory Automation (ETFA), pp. 1-4.

Krizhevsky, A., Sutskever, I. and Hinton, G. E., 2012. Imagenet classification with deep convolutional neural networks. In: F. Pereira, C. J. C. Burges, L. Bottou and K. Q. Weinberger (eds), Advances in Neural Information Processing Systems 25, Curran Associates, Inc., pp. 1097-1105.

Kumar, N., Belhumeur, P. N., Biswas, A., W.Jacobs, D., Kress, W. J., López, I. and Soares, J. V. B., 2012. Leafsnap: A computer vision system for automatic plant species identification. In: Proc. of the Europ. Conf. on Computer Vision (ECCV).

Latte, M. V., Anami, B. S. and B., K. V., 2015. A combined color and texture features based methodology for recognition of crop field image. International Journal of Signal Processing, Image Processing and Pattern Recognition 8(2), pp. 287-302.

LeCun, Y., Boser, B., Denker, J. S., Henderson, D., Howard, R. E., Hubbard, W. and Jackel, L. D., 1989. Backpropagation applied to handwritten zip code recognition. Neural Computation 1(4), pp. 541-551.

LeCun, Y., Bottou, L., Bengio, Y. and Haffner, P., 1998. Gradientbased learning applied to document recognition. In: Proceedings of the IEEE, pp. 2278-2324.
Lottes, P., Höferlin, M., Sander, S. and Stachniss, C., 2016a. Effective vision-based classification for separating sugar beets and weeds for precision farming. Journal on Field Robotics.

Lottes, P., Höferlin, M., Sander, S., Müter, M., SchulzeLammers, P. and Stachniss, C., 2016b. An effective classification system for separating sugar beets and weeds for precision farming applications. In: Proc. of the IEEE Int. Conf. on Robotics \& Automation (ICRA).

Lottes, P., Khanna, R., Pfeifer, J., Siegwart, R. and Stachniss, C., 2017. Uav-based crop and weed classification for smart farming. In: Proc. of the IEEE Int. Conf. on Robotics \& Automation (ICRA).

McCool, C., Perez, T. and Upcroft, B., 2017. Mixtures of lightweight deep convolutional neural networks: Applied to agricultural robotics. IEEE Robotics and Automation Letters.

Mortensen, A. K., Dyrmann, M., Karstoft, H., Jörgensen, R. N. and Gislum, R., 2016. Semantic segmentation of mixed crops using deep convolutional neural network. In: Proc. of the International Conf. of Agricultural Engineering (CIGR).

Perez-Ortiz, M., Peña, J. M., Gutierrez, P. A., Torres-Sánchez, J., Hervás-Martínez, C. and López-Granados, F., 2015. A semisupervised system for weed mapping in sunflower crops using unmanned aerial vehicles and a crop row detection method. Applied Soft Computing 37, pp. 533 - 544.

Perez-Ortiz, M., Peña, J. M., Gutierrez, P. A., Torres-Sánchez, J., Hervás-Martínez, C. and López-Granados, F., 2016. Selecting patterns and features for between- and within- crop-row weed mapping using uav-imagery. Expert Systems with Applications 47 , pp. $85-94$

Pfeifer, J., Khanna, R., Dragos, C., Popovic, M., Galceran, E., Kirchgessner, N., Walter, A., Siegwart, R. and Liebisch, F., 2016. Towards automatic uav data interpretation for precision farming. Proc. of the International Conf. of Agricultural Engineering (CIGR).

Potena, C., Nardi, D. and Pretto, A., 2016. Fast and accurate crop and weed identification with summarized train sets for precision agriculture. In: Proc. of Int. Conf. on Intelligent Autonomous Systems (IAS).

Rouse, Jr., J. W., Haas, R. H., Schell, J. A. and Deering, D. W., 1974. Monitoring Vegetation Systems in the Great Plains with Erts. NASA Special Publication 351, pp. 309.

Srivastava, N., Hinton, G., Krizhevsky, A., Sutskever, I. and Salakhutdinov, R., 2014. Dropout: A simple way to prevent neural networks from overfitting. Journal of Machine Learning Research 15, pp. 1929-1958.

Stachniss, C., Martínez-Mozos, O., Rottmann, A. and Burgard, W., 2005. Semantic labeling of places. In: Proc. of the Int. Symposium of Robotics Research (ISRR).

Szegedy, C., Ioffe, S. and Vanhoucke, V., 2016. Inception-v4, inception-resnet and the impact of residual connections on learning. Computing Research Repository.

Torres-Sanchez, J., López-Granados, F. and Peña, J., 2015. An automatic object-based method for optimal thresholding in uav images: Application for vegetation detection in herbaceous crops. Computers and Electronics in Agriculture 114, pp. 43 52.

Wang, X.-F., Huang, D., Du, J., Xu, H. and Heutte, L., 2008. Classification of plant leaf images with complicated background. Applied Mathematics and Computation 205, pp. 916 926. 